\title{
CASCADE CONTROL EXPERIMENTS OF RISER SLUG FLOW USING TOPSIDE MEASUREMENTS
}

\author{
Heidi Sivertsen * Sigurd Skogestad $*, 1$ \\ * Department of Chemical Engineering, Norwegian University \\ of Science and Technology, Trondheim, Norway
}

\begin{abstract}
Anti-slug control applied to two-phase flow provides a very challenging and important application for feedback control. It is important because it allows for operation that would otherwise be impossible, and challenging because of the presence of both RHP-poles and RHP-zeros.

To conduct experiments on pipeline-riser anti-slug control, a small-scale slug-loop has been build. The loop has been modelled and analyzed using the simplified riser-slug model by Storkaas. The aim has been to find ways to control the flow using only topside measurements, as subsea measurements can be unavailable offshore. The results from this analysis and experimental results using a cascade controller are presented in this paper. Copyright (c)2005 IFAC
\end{abstract}

Keywords: Cascade control, unstable, poles, feedback control, controllability analysis

\section{INTRODUCTION}

Riser slugging is a flow regime that can develop in multiphase production systems offshore. It is characterized by alternating bulks of liquid and gas caused by a low-point in the pipeline topography. This lowpoint is realized by a downsloping pipe terminating in a riser.

This flow regime can cause large and abrupt fluctuations in pipe pressure and gas and liquid flow rates at the outlet. Some of the problems associated with this flow are wear and tear on equipment and poor separation.

The behavior of pipeline-riser slug flow is illustrated in Figure 1. Liquid accumulates in the lowpoint of the riser, blocking the gas (1). As more gas and liquid enters the system, the pressure will increase and the riser will be filled with liquid (2). After a while the amount of gas that is blocked will be large enough to blow the liquid out of the riser (3). After the blow-out,

\footnotetext{
1 Author to whom correspondence should be adressed:
} skoge@chemeng.ntnu.no

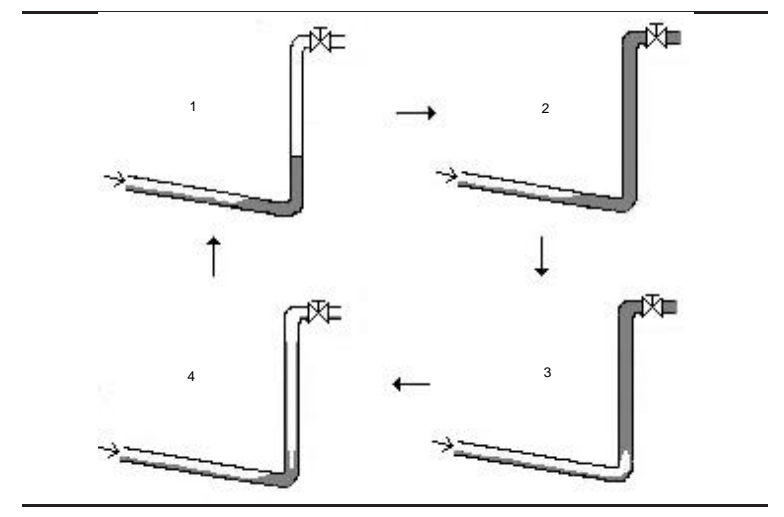

Fig. 1. Illustration of the cyclic behavior (slug flow) in pipeline-riser systems

a new liquid slug will start to form in the low-point (4).

Recently, anti-slug control has proven to successfully stabilize the flow at a pressure drop that would lead to slug flow if left uncontrolled. This has been done both in experiments (Hedne and Linga, 1990) and on offshore production facilities (Havre et al., 2000) and (Godhavn et al., 2005a). 
The above applications use subsea measurements, which are expensive to install and maintain and are sometimes unavailable. For this reason ways to control the flow using only topside measurements are sought.

A small-scale experiment (the Miniloop) has been build to test different control strategies. Use of a simple PI-controller with a subsea pressure measurement gave good results as expected from a controllability analysis of the system (Sivertsen and Skogestad, 2005). This analysis also indicated large problems using a single topside measurement for control.

In this paper, we consider combinations of topside measurements that might be able to stabilize the flow. One example of this is a cascade control configuration, where two topside measurements are combined.

\section{EXPERIMENTAL SETUP}

To test different control configurations, a small-scale two-phase flow loop with a pipeline-riser arrangement was build. The flow consists of water and air, which are mixed at the inlet of the system. Both the pipeline and the riser was made of a $20 \mathrm{~mm}$ diameter transparent rubber hose, which makes it easy to change the shape of the system. A schematic diagram of the test facilities is shown in Figure 2.

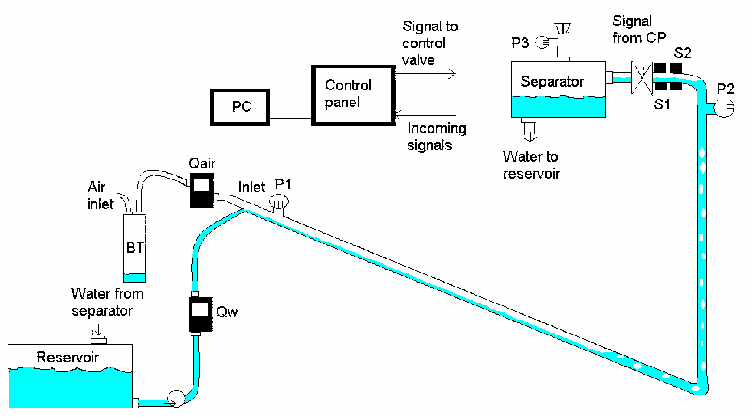

Fig. 2. Experimental setup

From the mixing point the flow goes trough the lowpoint at the bottom of the riser and depending on different conditions, slug flow may occur. At the top of the riser there is a separator, which leads the water to a reservoir. From there the water is pumped back into the system through the mixing point. The air is being let out through a small hole at the top of the separator.

For slugging to appear there must be enough air in the system to blow the water out of the 1,5 meter long riser. This requires a certain amount of volume, which is accounted for by a buffer tank (BT) between the air supply and the inlet. The volume of the gas can be changed by partially filling this tank with water.

The flow rates of gas (Qair) and water (Qw) determines whether we will have slug flow in open loop operation or not. These flow rates are measured at the inlet. Typically flow rates during an experiment are 1 $1 / \mathrm{min}\left(1.4 * 10^{-3} \mathrm{~kg} / \mathrm{min}\right)$ for the gas and $3 \mathrm{l} / \mathrm{min}(3$ $\mathrm{kg} / \mathrm{min}$ ) for the water.

A pressure sensor is located at the inlet (P1), and there are two pressure sensors located topside. One is placed at the top of the riser (P2) and one at top of the separator (P3). The latter is used for measuring the flow of air out of the separator.

There are two fiber optic sensors (S1, S2). These give a signal depending on the amount of water in the pipe. They can easily be mowed around to measure the hold-up at different locations in the loop.

A control valve is placed at the top of the riser. A signal from the control panel sets the opening percentage of the valve.

The control panel converts the analog signals from the sensors into digital signals. These signals are then sent to a computer. The signals are continuously displayed and treated using Labview software. Depending on the control configuration, some of the measurements are used by the controller to determine the opening percentage for the control valve.

\subsection{Flow estimates}

From the values given by the fiber-optic slug sensors the relative amount of water and air through the choke is found, which makes it possible to calculate the density of the mixture. The topside pressure sensor gives us the pressure drop over the control valve. Given pressure drop and density it is then possible to calculate the mass and volume flow through the choke, using a basic flow equation for volume flow (1).

$$
Q=C * z * \sqrt{\Delta P / \rho}
$$

The measurements from the fiber optic slug sensors needed some filtering because of spikes caused by reflections off the light on the water/air interface (Figure 3 ). Since these measurements where crucial for the flow estimates, the question was whether or not they had to be filtered too much for them to be used for control.

\section{CONTROLLABILITY ANALYSIS}

Storkaas et al. (2003) have developed a simplified model to describe the behavior of pipeline-riser slugging. The model is well suited for controller design and analysis. It consists of three states; the holdup of 


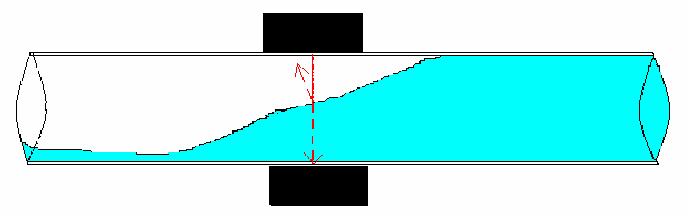

The tuned model can be used to perform a controllability analysis. This way we can predict which measurements are suitable for control, thus avoiding slug flow. The computed poles of the process at the valve position $Z=0.12$ and $Z=0.25$ are given in Table 1 .

Table 1. Poles of the system

\begin{tabular}{cc}
\hline \multicolumn{2}{c}{ Valve opening Z } \\
0.12 & 0.25 \\
\hline-20.3411 & -35.2145 \\
$-0.0197 \pm 0.1301 \mathrm{i}$ & $0.0071 \pm 0.1732 \mathrm{i}$ \\
\hline
\end{tabular}

Fig. 3. Reflection of light on water surface

gas in the feed section $\left(m_{G 1}\right)$, the holdup of gas in the riser $\left(m_{G 2}\right)$, and the holdup of liquid $\left(m_{L}\right)$. The model is illustrated by Figure 4. Using this model we are able to predict the variation of system properties such as pressure, densities and phase fractions.

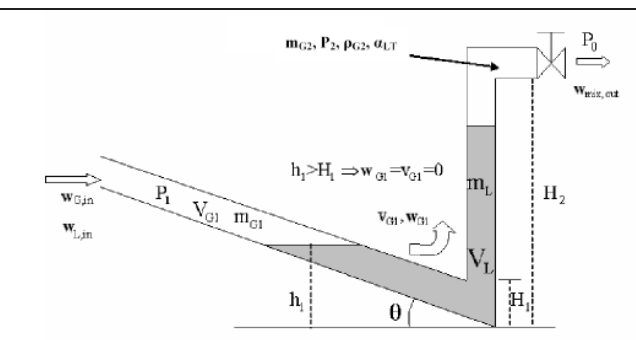

Fig. 4. Storkaas' pipeline-riser slug model

In order for the model to fit the MiniLoop, it needs to be tuned. To do this we compare the bifurcation diagrams of the model and the Miniloop. These diagrams are plotted in Figure 5. The upper lines shows the maximum pressure at a particular valve opening and the lower line shows the minimum pressure. The two lines meet at around $20 \%$ valve opening. This is the point with the highest valve opening which gives stable operation when no control is applied. When Storkaas' model is properly tuned, the bifurcation point from the model will match the one from the experimental data. The dotted line in the middle shows the unstable steady-state solution. This is the desired operating line with closed-loop operation. Details about the tuned model are given by (Storkaas, 2005).
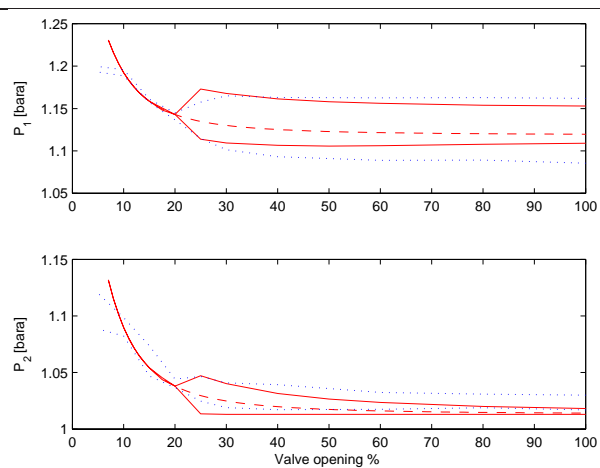

Fig. 5. Bifurcation diagrams from experimental data (dotted line) and Storkaas' model (solid line)

A valve opening of $12 \%$ results in only LHP poles, so running open loop with this valve opening will lead to stable flow in the pipeline. However, when the valve opening is set to $25 \%$ a pair of RHP poles indicates an unstable system.

This means that riser slugging will occur when the system is run open loop with this valve opening. These results could also be predicted from the bifurcation diagram in Figure 5, where we see that the system goes unstable for valve openings larger than $20 \%$.

To stabilize the flow the measured topside pressure, $P_{2}$, and the estimated topside density $\rho$, volume flow $F_{q}$ and mass flow $F_{w}$ are available. The upstream pressure measurement $P_{1}$ is also available.

Unstable poles need feedback for stabilization, and place a lower bound on the bandwidth of the feedback system. It is well known that stabilization (shifting of poles from RHP to LHP) is fundamentally difficult if the plant has a RHP-zero close to a RHP-pole $p$. Skogestad and Postlethwaite (1996) derives the approximate lower bandwidth limit $w_{c}>1.15|p|$ for imaginary RHP poles of magnitude $|p|$. For a real RHP zero $z$ they derive an upper bandwidth limit $w_{c}<z / 2$. From the $25 \%$ valve opening we see that all RHP zeros need to be larger than approximately 0.4 to be able to stabilize the system.

To find suitable measurements for control, the openloop zeros for the system need to be inspected. They are given in Table 2 .

Table 2. Open loop zeros of the system for different measurement alternatives

\begin{tabular}{ccccc}
\hline$P_{1}$ & $P_{2}$ & $\rho$ & $F_{q}$ & $F_{w}$ \\
\hline-1.285 & 46.984 & 0.092 & -3.958 & -65.587 \\
& 0.212 & -0.0547 & $-0.369 \pm 0.192 \mathrm{i}$ & $-0.007 \pm 0.076 \mathrm{i}$ \\
\hline
\end{tabular}

Because of the bandwidth limitations it seems evident that measurements $\rho$ or $P_{2}$ cannot be used for stabilization. Measurement $P_{1}$ does not have any RHP zeros, so no particular problems using this measurement are to be expected. Also $F_{q}$ and $F_{w}$ could be used for stabilization. However the zeros close to the origin indicates a steady-state gain close to zero, so good control performance cannot be expected. The steady-state gain for $F_{q}$ and $F_{w}$ are found to be 0.4545 and 0.0015 respectively. Using these measurements we may be able to stabilize the system, but we cannot 
affect its steady-state behavior and the system will "drift".

From the controllability analysis we can draw the conclusion that when using only a single measurement for control, the inlet pressure $P_{1}$ is the only suitable choice.

However, in many cases only topside measurements are available, and even though none of the topside measurements can be used as a single measurement, there is still the possibility that combinations of these measurements can stabilize the flow. The reason is that systems with extra outputs generally have no zeros, except when the same RHP-zero appears in all outputs. Table 2 shows that this is not the case here.

One possibility is to use a cascade control configuration with the flow measurements $F_{q}$ or $F_{w}$ in the inner loop and $P_{2}$ as measurement for the outer loop. The outer loop will then take care of the low-frequency performance in the system, while the flow measurement are used only for stabilization.

\section{EXPERIMENTAL RESULTS}

\subsection{PI-controller}

The above analysis showed that the inlet pressure, $P_{1}$, was the only suitable choice when using a single measurement for control. Experiments on the lab confirm that a PI-controller (Figure 6) using this measurement stabilizes the flow. The controller has a setpoint of 1.128 bara, gain $K=-2.5$ bara $^{-1}$ and integral time $\tau_{i}=10 \mathrm{~s}$.

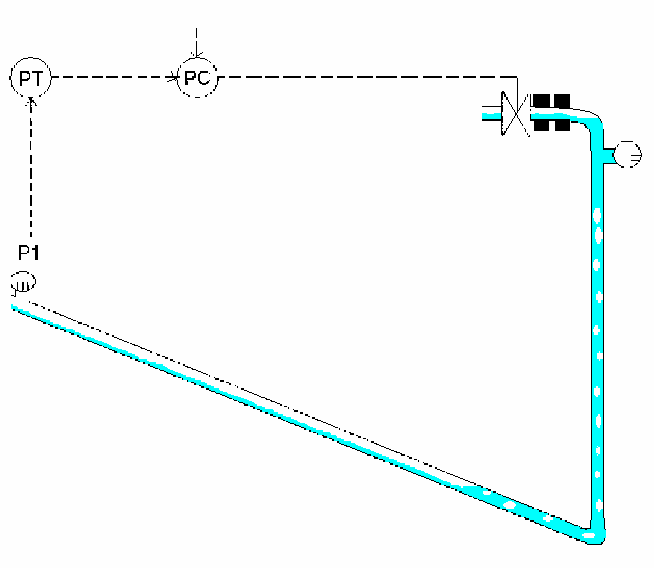

Fig. 6. PI control using measurement $P_{1}$

Figure 7 shows how the flow is stabilized after starting from open loop with a valve opening of $30 \%$. It remains stable for the 6 minutes the controller is active. The simulated values using Storkaas' model are shown in the lower plot.

We see that the controller efficiently stabilizes the flow, confirming the results from the controllability
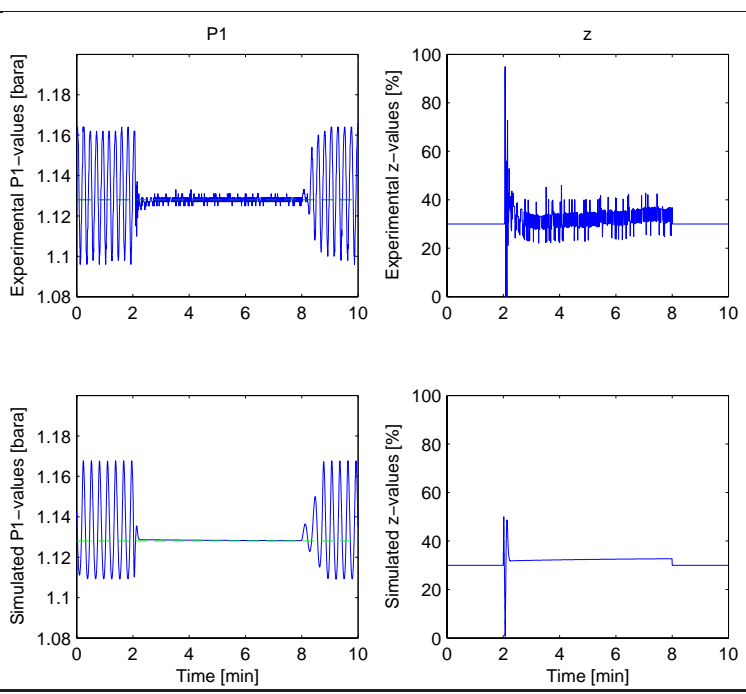

Fig. 7. Experimental (upper plots) and simulated (lower plots) results using a PI-controller

analysis. However, this measurement can be unavailable in offshore installations because of its subsea location.

\subsection{Cascade controller}

To avoid the need for subsea measuremens, a cascade configuration using the topside pressure, $P_{2}$, in the outer loop and the mass flow, $F_{w}$, in the inner loop has been investigated. The control configuration is illustrated in Figure 8.

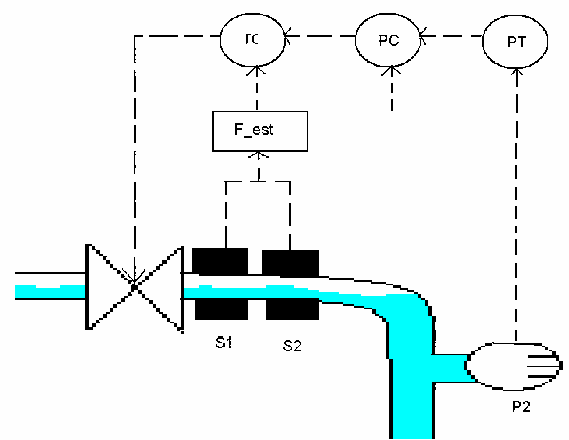

Fig. 8. Cascade control using measurements $P_{2}$ and $F_{w}$

Storkaas and Skogestad (2003) have proved theoretically that this works for another case of riser slugging and it has also been shown that it is possible to stabilize the flow this way on larger test facilities (Godhavn et al., 2005b). However, so far we have not been able to verify this experimentally with the Miniloop. On the other hand simulations of the Miniloop confirme that this should be possible, as Figure 9 shows. The tuning parameters for the PI-controller in the outer loop are $K=-0.05$ bara $^{-1}$ and $\tau_{i}=30 \mathrm{~s}$. The setpoint is 1.030bara. The inner loop consisted of a P-controller with gain $K=10 \mathrm{~s} / \mathrm{kg}$. 

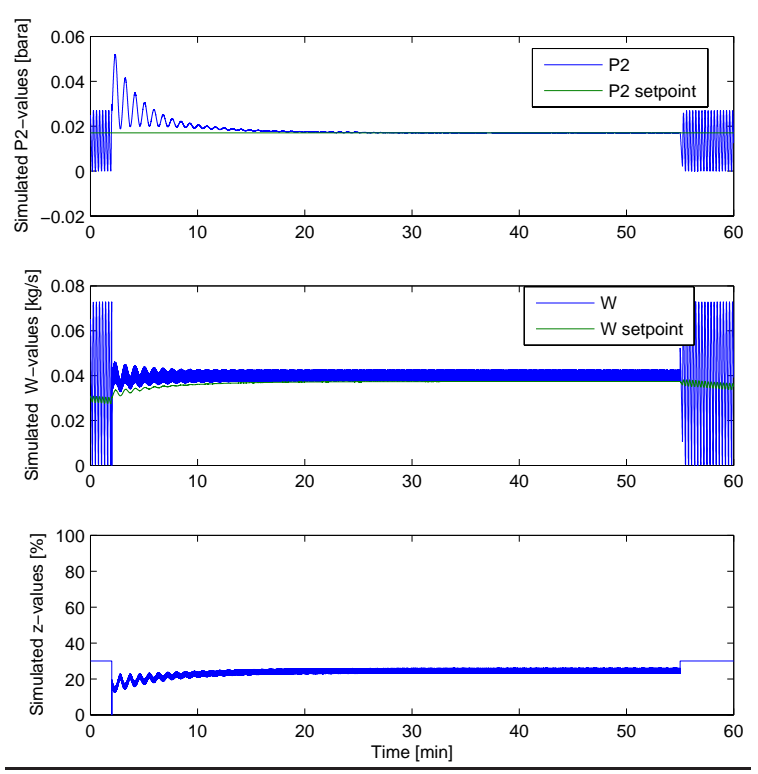

Fig. 9. Simulated values using a cascade controller

We see it takes the controller much more time to stabilize the flow than it did with the PI-controller which stabilized the flow almost immediately. Storkaas (2005) shows that this is due to unstable (RHP) zeros for the model as seen from the outer loop, when the inner loop is closed. These instable zeros will limit the bandwith of the system, and the controller based on only topside measurements are therefore slower than control systems based on upstream pressure measurements.

As mentioned, the controller did not work as well in the lab as it did for the simulations. The flow measurement was estimated from the signals given by the fiber-optic slug sensors, and it turned out that they had to be filtered to such an extent that they could not be used to stabilize the system at the lab.

\subsection{Multivariable controller}

It has earlier been shown that a MISO (Multiple Input - Single Output) $\mathcal{H}_{\infty}$ based on the same measurements as the cascade control configuration described above could give better performance when applied on riserslugging than the cascade controller (Storkaas, 2005).

MISO controllers are more difficult to tune online than cascade controllers, and direct model-based controller design is usually needed. This can be done using Storkaas' simplified model in the case of the Miniloop. The method involves finding the controller $\mathrm{K}$ that minimizes the maximum singular value $\left(\mathcal{H}_{\infty}\right.$ norm) of one or more weighted closed loop transfer functions and thereby maximizing robustness and, trough the weights, shaping the closed loop response.

\section{CONCLUSION}

From the controllability analysis it was found that using the inlet pressure was the only measurement that could be used as single measurement for antislug control. If only topside measurements where to be used, combinations of at least two of them was necessary.

A cascade controller was tested out, using the topside pressure and estimated mass flow as measurements. It was first tested in simulations using a simplified riser-slug model. The results showed that the cascade controller managed to stabilize the flow.

When trying the cascade controller in experiments on a small two-phase loop, the results where not so good. The reason for this was that the volume flow was estimated using hold up measurements that had to be heavily filtered.

If better measurements for the hold-up in the pipeline was available it could still be possible that the cascade configuration would give better results. Instruments based on capacitance would probably have been a better choice instead of the fiber optic slug sensors used in the experiments described in this paper. Also other, more robust, control configurations might handle the problem better than the cascade configuration. One example of such is the $\mathcal{H}_{\infty}$ controller which we plan to test in later experiments.

\section{ACKNOWLEDGMENTS}

The authors would like to thank former M.Sc. students Ingvald Baardsen and Morten Søndrol for assisting in building the Miniloop and performing experiments. Former Ph.D. student Espen Storkaas deserves thanks for help using his model. Also we would like to thank Statoil and the Norwegian Research Council for financial support.

\section{REFERENCES}

Godhavn, J.-M., S. Strand and G. Skofteland (2005a). Increased oil production by advanced control of receiving facilities. IFAC'16, Prague, Czech Republic.

Godhavn, J.M., M.P. Fard and P.H. Fuchs (2005b). New slug control strategies, tuning rules and experimental results. Journal of Process Control (15), 547-577.

Havre, K., K.O. Stornes and H. Stray (2000). Taming slug flow in pipelines. ABB review 4, 55-63.

Hedne, P. and H. Linga (1990). Supression of terrein slugging with automatic and manual riser choking. Advances in Gas-Liquid Flows pp. 453-469.

Sivertsen, H. and S. Skogestad (2005). Anti-slug control experiments on a small scale two-phase loop. ESCAPE'15, Barcelona, Spain. 
Skogestad, S. and I. Postlethwaite (1996). Multivariable Feedback Control. John Wiley \& Sons.

Storkaas, E. (2005). Stabilizing control and controllability: Control solutions to avoid slug flow in pipeline-riser systems. PhD thesis. Norwegian University of Science and Technology.

Storkaas, E. and S. Skogestad (2003). Cascade control of unstable systems with application to stabilization of slug flow. AdChem'03 Hong Kong.

Storkaas, E., S. Skogestad and J.M. Godhavn (2003). A low-dimentional model of severe slugging for controller design and analysis. In: Proc. of MultiPhase '03, San Remo, Italy, 11-13 June 2003. 